\title{
Un estudio preliminar sobre la recepción de las campañas de violencia de género televisivas del Ministerio de Sanidad, Política Social e Igualdad (2008-2011)
}

\author{
Dr. Emma CAMARERO CALANDRIA \\ Universidad de Salamanca \\ emma.camarero@usal.es
}

Dda. María MARCOS RAMOS

Universidad de Salamanca

mariamarcos@usal.es

Recibido: 5.05.2012

Aceptado: 15.11.2012

\section{RESUMEN}

En el presente artículo se dan a conocer los primeros resultados obtenidos del estudio piloto realizado sobre las campañas de violencia de género televisivas realizadas por el Ministerio de Sanidad, Igualdad y Políticas Sociales en el período 2008-2011. La investigación tiene como objetivo principal analizar la percepción de los ciudadanos y ciudadanas sobre estas campañas de prevención de violencia de género. Especialmente se trata de saber si son instrumentos eficaces y realmente sensibilizadores en la lucha contra la violencia de género o por el contrario, los medios utilizados en estas campañas resultan ineficaces o insuficientes. Este estudio previo trata también de establecer la validez del cuestionario utilizado, una vez estudiados los resultados obtenidos en esta primera fase de la investigación. Tras su análisis, dichos resultados serán el germen de un macroestudio posterior donde se ampliará la muestra de esta investigación sobre las campañas televisivas contra la violencia de género en España.

Palabras clave: violencia de género, televisión, campaña publicitaria, investigación cuantitativa.

\section{A preliminary study on the receipt of the television campaigns about violence of gender realized by the Ministry of Health, Social Politics and Equality (2008-2011).}

\begin{abstract}
This article shows the early results of the pilot research about gender violence TV campaigns promoted by the spanish Ministry of Health, Gender and Social Policies. The mentioned research has the main purpose of analyze the citizenship perception about the prevention campaigns in social media and their effectiveness in the fight against gender violence. This previous survey is the seed of an extensive research project about media campaigns of gender violence prevention in Spain. Its first data intended to be the pattern of the completely developed research. The final intent, after consider all the results, is to reflect about the real awareness capability and their social usefulness.
\end{abstract}


Keywords: Gender violence; television advertising campaign; quantitative research.

\section{INTRODUCCIÓN}

La violencia de género es un desgraciado fenómeno social que se da en mayor o menor medida y frecuencia en todas las sociedades del mundo. El papel absolutamente secundario, cuando no marginal de la mujer en gran parte del mundo agudiza esta situación. El grado de desarrollo de cada sociedad, el rol alcanzado por la mujer respecto al hombre, los planes educativos y la fluctuación de este fenómeno, son factores que entre otros muchos obligan a variar los contenidos y mensajes de las campañas contra la violencia de género. Más aún cuando hablamos de campañas enfocadas al medio televisivo.

Esta investigación se ocupa de estudiar por un lado, los elementos que configuran a nivel audiovisual, lingüístico y simbólico el lenguaje de estas campañas, y relacionar dichos elementos con la percepción que la sociedad española tiene de estas formas mediáticas de sensibilización.

La segunda parte de este trabajo tiene como objetivo analizar la recepción por parte del público de las campañas realizadas por el Ministerio de Sanidad, Servicios Sociales e Igualdad en el período 2008-2011. Para poder conocer la opinión de los receptores se diseñó un cuestionario en el que se preguntaba sobre diversos temas relacionados con la violencia de género y con las diferentes campañas realizadas por el Gobierno desde el 2008 a 2011.

\section{ANÁLISIS DE LAS CAMPAÑAS CONTRA LA VIOLENCIA DE GÉNERO EN TELEVISIÓN DEL MINISTERIO DE IGUALDAD DE ESPAÑA (2008-2011)}

En sus inicios, la imagen de la mayoría de las campañas ha sido la cara de una mujer con las huellas de la violencia, una imagen que incidía sobre todo en la idea de que éste era un problema que sólo afectaba a las mujeres. El lenguaje textual también estaba al servicio de este objetivo. En su momento, fue importante destacar esta situación ante la invisibilidad social de esta violencia debido al desconocimiento de su realidad. Pero además, se considera imprescindible en cualquier caso, "realizar un acercamiento desde la perspectiva estadística que permita efectuar un seguimiento de la magnitud y la evolución del fenómeno al menos en algunas de sus manifestaciones" (II Informe Anual sobre Violencia de Género, 2009: 3). No es una violencia abstracta ni genérica, ya que afecta a la mujer, pero también a los hijos, a la familia, al entorno social, y, evidentemente, la violencia que ejerce el maltratador no debe tolerarse y por ello es él quien debe recibir toda la crítica y reprobación social- 
Al eslogan, que es por definición la frase más destacada de la campaña publicitaria, además de brevedad se le exige ciertas cualidades de memorización y una total especificidad. A veces es una síntesis -formal o de contenido-, del texto que la acompaña. En las campañas del Ministerio de Igualdad, los eslóganes elegidos son realmente los que deciden el resto del lenguaje de cada spot. Por tanto son la consecuencia de los hechos narrados, en definitiva la ansiada, pero, tan sólo teórica, solución al problema.

En cuanto a los elementos analizados desde una metodología cualitativa, la iconización forma parte de las estrategias generales de la persuasión, pero aún asumiendo sus valiosas aportaciones a este trabajo, se pretende además "situar el fenómeno de la textualidad publicitaria en el plano perceptivo-comunicativo" (Vellón, 2007: 31), ya que contextualiza metodológicamente las líneas interpretativas de este estudio.

Para la realización de esta parte del estudio hemos utilizado la siguiente metodología:

- Exhaustiva búsqueda documental, videográfica y bibliográfica relativa a las campañas contra la violencia de género para televisión con tres niveles de aplicación: autonómico, nacional e internacional.

Análisis de las campañas concretas a las que se refiere este estudio, las realizadas por el Ministerio de Igualdad entre 2008 y 2011, desde su puesta en marcha hasta su finalización. Se analiza para ello la documentación relativa a estas campañas, tanto en su realización audiovisual como en sus contenidos, objetivos, ámbito de aplicación, temporalidad, presupuestos y recursos materiales.

- Estudio de los datos cuantitativos y cualitativos relativos a la violencia de género en España en el período de vigencia de cada una de las campañas, así como datos estadísticos relacionados con la aplicación y puesta en marcha de las campañas en televisión. Principalmente, se han analizado cifras oficiales de carácter estadístico facilitadas por, entre otros organismos, el Ministerio de Sanidad, Igualdad y Políticas Sociales, el Observatorio de la Mujer o en Centro de Investigaciones Sociológicas (CIS).

- Estudio comparativo de los datos obtenidos a través del cuestionario para saber de qué forma las campañas analizadas son percibidas por los receptores.

Se han analizado para esta muestra las campañas en televisión contra la violencia de género realizadas por el Ministerio de Igualdad (actualmente Ministerio de Sanidad, Igualdad y Políticas Sociales) durante los años 2008, 2009, 2010 y 2011. Nuestro estudio parte de la campaña 2008 por ser la primera vez que el Ministerio de 
Igualdad implementa una campaña continuada y multifocal contra la violencia de género en España. A estas actuaciones ministeriales habrían de unirse las realizadas por gobiernos autonómicos, ayuntamientos e instituciones, valorables en su conjunto en la lucha contra la violencia de género.

En el lenguaje publicitario, el mensaje, suele coincidir plenamente con las campañas llevadas a cabo por el Ministerio cuando no lo complementan.

"Las campañas contra los malos tratos pueden adoptar diferentes formas en su propósito de prevenir, anticipar, combatir y, finalmente, erradicar la violencia contra las mujeres. En este objetivo múltiple se encuentra la motivación central que guía la idea de hacer campañas de prevención contra este tipo de violencia. Las campañas responden a un determinado análisis del problema de los malos tratos e intentan contrarrestar algunas de las causas que los posibilitan o de neutralizar algunos de los obstáculos que se oponen a su erradicación” (Molina González, 2005: 11).

Aunque en la actualidad cada vez más campañas son evaluadas de forma sistemática, esta estrategia aún no se ha convertido en una práctica habitual. En general, se analizan los datos mensuales y anuales de violencia de género pero rara vez se reflexiona y analiza la eficacia de las campañas televisivas en el mayor o menor éxito en la lucha contra la erradicación de este fenómeno social negativo. Evidentemente, la capacidad de concienciación y sensibilización de las campañas en televisión es limitada, debido a una serie de causas y circunstancias sociales que fluctúan en el tiempo, cambian y hacen de este fenómeno, un problema con numerosas vertientes causales.

La enorme complejidad de la violencia de género, sus diversos orígenes y desarrollo, dificulta enormemente su estudio y la individualización de sus causas. Por ello, los primeros resultados de este estudio apuntan a que aún no se ha conseguido encontrar la vía correcta para lograr la máxima sensibilización a través del uso de campañas publicitarias en televisión.

\subsection{CAMPAÑA 2008: “ANTE EL MALTRATADOR, TOLERANCIA CERO”.}

En 2008 - cuatro años después de la aprobación de la Ley Integral ${ }^{1}$ (Ley Orgánica 1/2004) para la lucha contra la Violencia de Género-, aparece en España el primer plan de comunicación sostenido en el tiempo que incluían campañas de sensibilización en televisión, y que buscaba varios objetivos: la complicidad de toda la sociedad para erradicar la violencia de género; informar a las víctimas de sus derechos y de los instrumentos previstos para su protección, y conseguir la

\footnotetext{
${ }^{1}$ Esta Ley Orgánica 1/2004, de 28 de diciembre, de Medidas de Protección Integral contra la Violencia de Género, se puede consultar en: http://www.boe.es/boe/dias/2004/12/29/pdfs/A42166-42197.pdf.
} 
desaprobación del conjunto de la sociedad hacia los maltratadores.

La ovedad de esta campaña radica en buscar la connivencia de los hombres en el rechazo contra la violencia de género y especialmente contra los maltratadores, mostrar las consecuencias de la violencia en los menores y la prevención de la violencia en mujeres, con spots protagonizados por españolas e inmigrantes.

Si tenemos en cuenta que el eslogan de esta campaña, "Contra el maltratador, tolerancia cero", había sido creado once años antes en el Parlamento Europeo, podemos entender que el estadio de desarrollo de la conciencia colectiva española había necesitado de un tiempo para llegar a un grado de madurez suficiente como para aceptar este tipo de mensajes textuales.

El spot protagonizado por los hombres ${ }^{2}$ utiliza un lenguaje que evidencia el
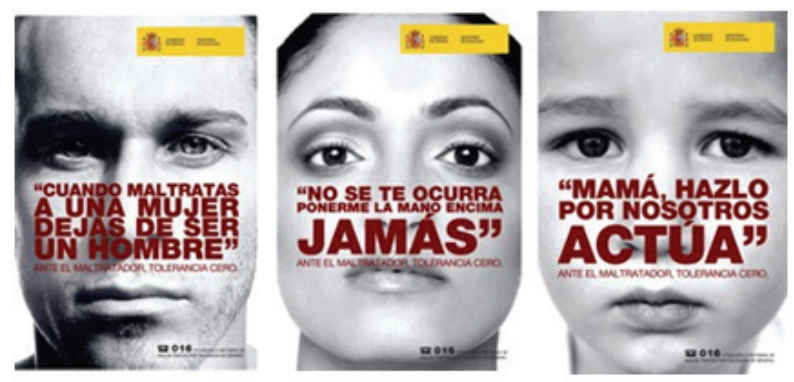

rechazo del resto de los hombres al agresor - "cada vez que maltratas a una mujer, dejas de ser un hombre"-, mientras que el spot protagonizado por las mujeres las destaca con la voz y la iniciativa de decir no al violento en nombre de toda la sociedad, "visto como quiero, no tengo miedo, no se te ocurra levantarme la mano jamás". El spot incluye la presencia de mujeres inmigrantes por la necesidad de que los mensajes de estas campañas empiecen a penetrar con urgencia en la comunidad inmigrante . $^{3}$

Esta campaña pretende además, que los menores sean los protagonistas de un

\footnotetext{
${ }^{2}$ La campaña está compuesta por 3 spots, estando protagonizado cada uno de ellos por un grupo de población. De este modo, hay un spot protagonizado por mujeres, otro por niños y el último, por hombres.

${ }^{3}$ Los datos sobre violencia de género de 2008 en España confirman que el 36,8 \% de los agresores y el $43,4 \%$ de las víctimas eran extranjeras. En 2003, los agresores y víctimas extranjeras eran solo el $15,5 \%$ y el $12,7 \%$ respectivamente, lo que supone un aumento de más del 30\% en cinco años (datos del Ministerio de Sanidad, Política Social e Igualdad). Hay que señalar que el porcentaje de población inmigrante creció en esos cinco años en España en cinco puntos porcentuales pasando de un 6,24\% en 2003 a un $11,3 \%$ en 2008 , teniendo en cuenta solo a los inmigrantes censados.
} 
futuro sin violencia de género. En este caso, junto al eslogan general de la campaña aparece otro de profundo significado, "no lo hagas por nosotros, hazlo por nosotros". Se trata de incidir en uno de los aspectos de este drama -los hijos-, para que la sociedad analice las consecuencias que en terceras personas tiene la violencia de género. Mostrando el daño que puede producir directamente a los menores, el spot protagonizado por los hijos de las víctimas pretende provocar un rechazo social más profundo y radical hacia estas conductas.

Esta campaña utiliza un lenguaje directo, enunciado de forma imperativa, dirigido al hombre o la mujer como simbólicos interlocutores.

A pesar de ser la primera campaña global realizada en España, su puesta en marcha no significó un avance significativo en la lucha contra la violencia de género. El año 2008 ha sido el de mayor número de muertes por violencia de género de la última década. Dado que el eslogan y la propia campaña de concienciación no lograron una penetración social relevante, desde el Ministerio de Igualdad se decidió empezar a trabajar en otra dirección, utilizando un lenguaje más conciliador y menos agresivo en campañas posteriores.

\subsection{CAMPAÑA 2009: “ANTE EL MALTRATO, TODAS Y TODOS A UNA”.}
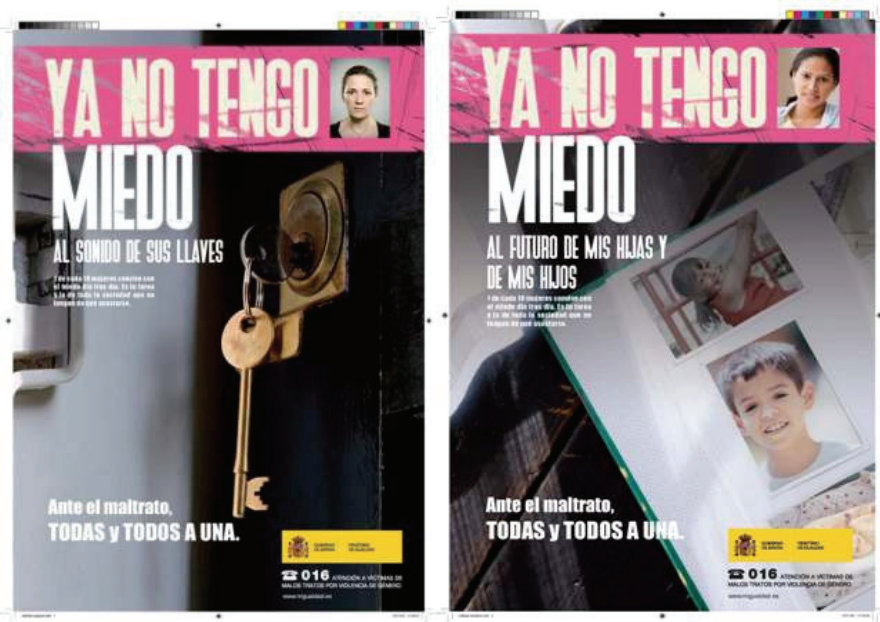


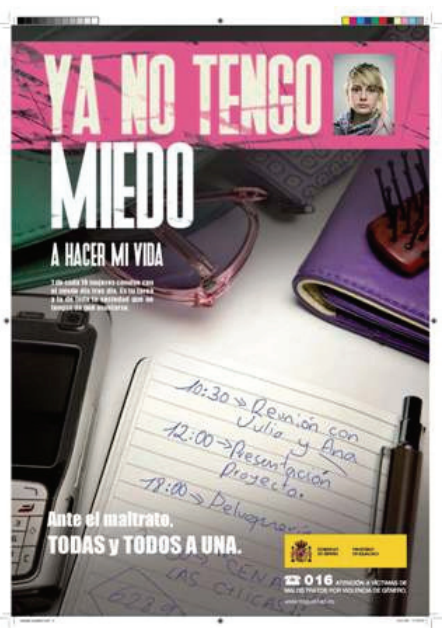

La campaña institucional realizada por el Ministerio en el año 2009, vuelve a utilizar parte del eslogan de la anterior, "ante el maltratador...", pero en cambio, la segunda parte del lema conlleva un mensaje mucho más conciliador que en 2008, "todos y todas a una". El lenguaje textual utilizado es mucho más escueto, transmitiendo la situación de temor y miedo en la que viven las mujeres víctimas de violencia de género con una llamada a la sociedad para que actúe frente a los malos tratos. Así, la campaña utiliza el mensaje en positivo: "ya no tengo miedo", convirtiéndose en la respuesta de las mujeres al sentir que toda la sociedad está detrás de ellas para protegerlas y apoyarlas.

Esta campaña marca una ruptura más que una evolución con la campaña anterior. Frente al imperativo, a la imposición, a colocar a la mujer ante la necesidad de tomar una decisión y de enfrentarse a sus propios miedos de forma individual, el mensaje utilizado en 2009 incide en la idea de que las mujeres no están solas para tomar esas decisiones.

Este cambio de lenguaje es producto en gran medida de un dato estadístico de 2008; solo el $23,7 \%$ de las víctimas mortales había denunciado malos tratos, y de ellas, el 2,6\% había retirado la denuncia. Dada la dificultad para convencer a muchas mujeres de que denuncien, el eslogan de esta campaña busca la complicidad de la sociedad para ayudarlas.

Esta campaña publicitaria, junto a otra serie de medidas puestas en marcha en 2009 en la lucha contra la violencia de género, unida a una serie de circunstancias de carácter coyuntural, consiguieron que ese año la tasa de víctimas mortales por violencia de género fuera la más baja de los últimos 20 años, pasando de 76 a 55 
asesinatos, un 27,6\% menos que en 2008 .

\subsection{CAMPAÑA 2010: "SACA TARJETA ROJA AL MALTRATADOR”.}

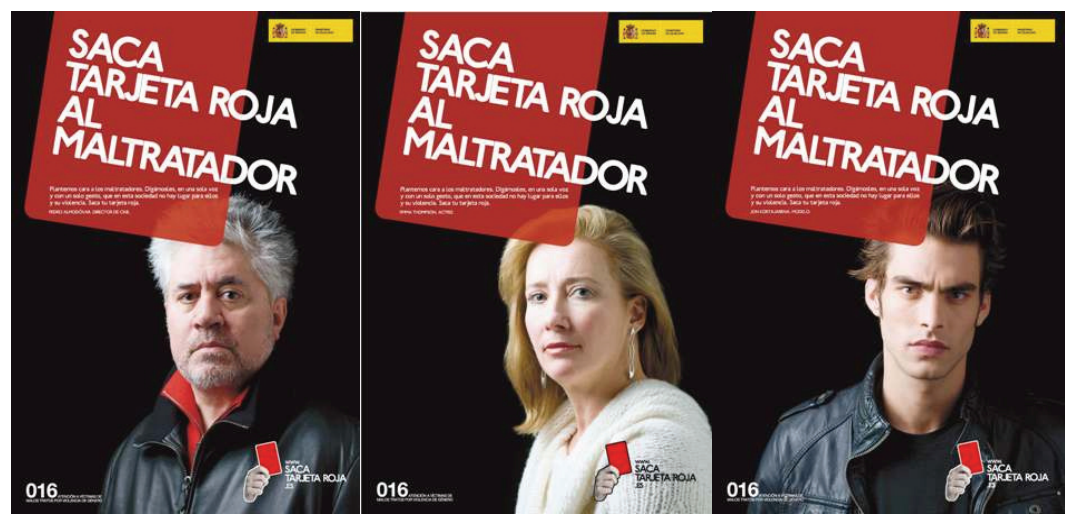

Con los datos positivos relativos a la lucha contra la violencia de género del 2009, en 2010 se crea la más ambiciosa y amplia campaña realizada hasta el momento por el Ministerio. Si en 2008 el presupuesto de estas campañas ascendió a 4,3 millones de euros, para el 2010 en Ministerio de Igualdad destinó casi el doble, unos 8,5 millones. Esta campaña contará con la presencia de personajes públicos como protagonistas.

El símbolo que preside la campaña en 2010 es la tarjeta roja como reflejo del rechazo social contra el maltratador y por ser un gesto y un objeto de significado ampliamente conocido, particularmente en el ámbito deportivo. Quien no juega limpio, queda fuera de la sociedad. De este modo, cada personaje que muestre la tarjeta roja reflejará el rechazo a los maltratadores y, al mismo tiempo, el apoyo a las mujeres que sufren su violencia.

No puede negarse que existe un grado de oportunismo en este eslogan. El argot futbolístico está de plena actualidad en 2010 en España, ya que tiene lugar el Mundial de Fútbol de Sudáfrica con la selección española como una de las favoritas. Esta campaña, utilizando una cierta banalización del lenguaje, es en cambio, la más compleja en cuanto a contenidos, difusión, medios publicitarios y recursos de todas las creadas hasta ese momento por el Ministerio de Igualdad.

La campaña original para televisión y cine está compuesta por ocho spots de entre 20 y 120 segundos. El objetivo es generar un movimiento social, de forma que cada uno de nosotros, a título personal, acoja el símbolo de la Tarjeta Roja al maltratador y lo haga suyo. Para ello se crea una página web www.sacatarjetaroja.es en la que 
quien lo desee puede descargar su propia tarjeta roja, hacerse una foto con ella y subirla a la misma web, como símbolo de adhesión a la campaña.

El lenguaje utilizado en esta campaña es una simbiosis en el plano simbólico del mensaje de las dos campañas anteriores. De hecho, la simplicidad de la imagen utilizada, actores contra fondos oscuros débilmente iluminados, otorga al lenguaje textual un valor esencial en la transmisión del mensaje de la campaña. Alguno de los spots -especialmente los realizados para el medio televisivo, los más cortos de duración-, cuentan con la participación de personajes famosos, y utilizan de nuevo el lenguaje imperativo. Los personajes, hombres en su mayoría, se dirigen a la mujer de forma negativa, e incluso amenazante: "tú es que no sabes hacer nada bien, me vas a llevar a la ruina, ¿no sabes más que gastar?". Cuando es la mujer no maltratada la protagonista, se dirige de forma directa al maltratador: "claro que puedes pegarle, claro que gritas más fuerte, claro que llama a escondidas". Los spots finalizan con un mensaje proclamado por hombres a otros hombres: "quien maltrata a una mujer no tiene sitio en nuestra sociedad. Plántate, sácale tarjeta roja al maltratador".

Los spots de más larga duración representan personajes que pretenden ser reflejo de muchas personas que de forma cercana, pero indirecta y con sentimiento de culpa, han vivido el drama del maltrato: un hijo, un hermano, un amigo. El lenguaje utilizado en este caso busca, a través de la utilización de frases muy habituales, generar conciencia sobre la necesidad de actuar ante el maltrato: "él siempre hablaba mal de ella" "yo se lo dije, que se pasaba un huevo"; "es duro porque es tu padre y le odias"; "son cosas de ellos, de mi hermano, decía yo"; "no piensas tampoco que va a llegar tan lejos"; "yo la vi justo el día antes de que la matara"; "si no hubiera estado sola"; "tenía que haber ido a por él, a la policía, yo qué sé"...

El final del spot representa a esos mismos personajes con un papel activo en cada historia de maltrato: "estoy harto de escuchar que tú no querías. Si golpeas, amenazas o insultas, no cuentes con mi silencio porque voy a denunciarte". De nuevo un lenguaje imperativo, de enfrentamiento al agresor, pero esta vez, a diferencia de la campaña de 2008, no es la mujer la que se enfrenta directamente, sino son los hombres, iguales ante los ojos el maltratador, los que toman la iniciativa de la sociedad contra la violencia de género.

A pesar de la enorme cantidad de medios, canales y recursos sin precedentes al servicio de esta campaña y, en general, al servicio de la lucha contra la violencia de género, ateniéndonos a las cifras de víctimas mortales, no parece que esta campaña las medidas puestas en marcha contra la violencia de género en 2010 hayan conseguido los objetivos marcados. En 2010 fueron asesinadas 73 mujeres, lo que supone un aumento del $23,3 \%$ con respecto a 2009. 


\subsection{CAMPAÑA 2011: "NO TE SALTES LAS SEÑALES, ELIGE VIVIR".}

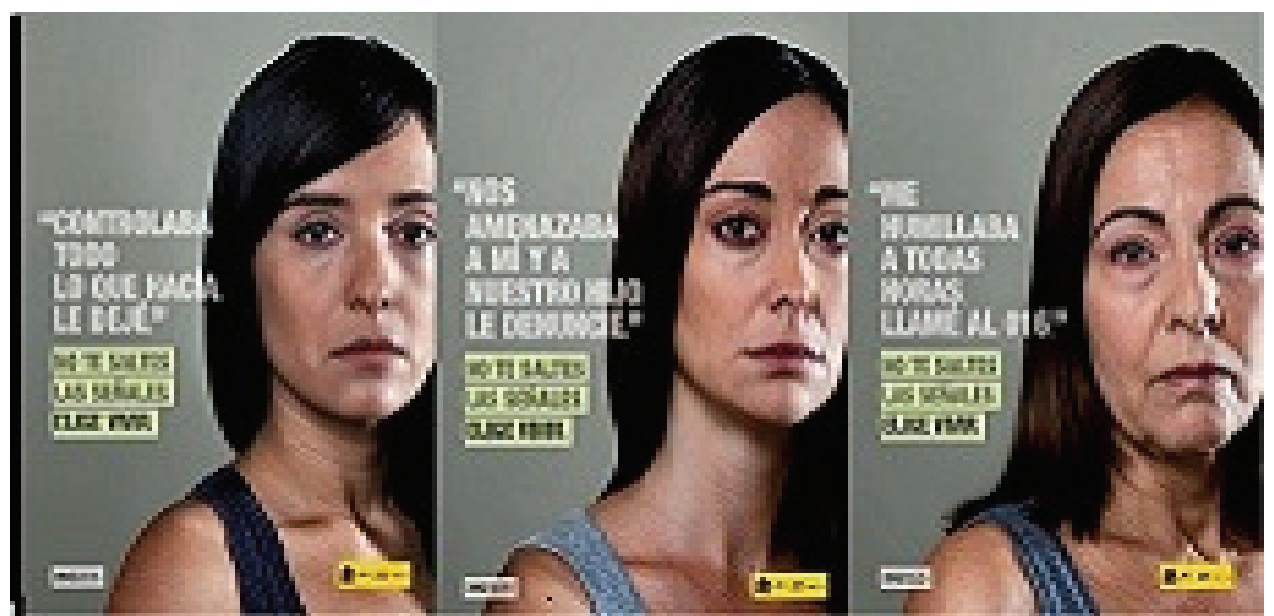

En esta campaña, presentada en septiembre de 2011, el mensaje está dirigido directamente a las mujeres--buceando en el origen del problema y en las señales de un posible maltrato futuro. La prevención a través de la sensibilización, constituye una de las claves en la lucha contra la violencia de género. Esta campaña busca de nuevo la complicidad de toda la sociedad para erradicar la violencia contra las mujeres, informar a las víctimas de sus derechos y de los instrumentos previstos para su protección, y conseguir el rechazo social hacia los maltratadores. La campaña advierte, ante este tipo de señales, a través de un mensaje dirigido a las mujeres víctimas y también a sus entornos, del daño irreversible de la violencia de género.

La campaña tiene un presupuesto de algo más de 4,5 millones de euros, para la planificación de medios y una duración continuada y multifocal durante todo un año. Ello supone una reducción bastante considerable de fondos con respecto a 2010. Esta campaña se crea para ser emitida en todas las cadenas de televisión y radios nacionales, así como en las principales autonómicas, cada una en su lengua específica. Además, se programa en las emisoras de radiofórmula, lo que es una novedad con respecto a las campañas anteriores.

Sin embargo, no presenta casi novedades desde el punto de vista del tratamiento del mensaje. Sigue obviándose la violencia explícita, para mostrar en cambio las consecuencias y repercusiones de la misma en nuestro entorno. Aunque hay referencias al maltratador, esta vez no es el objetivo del mensaje.

Los datos estadísticos de 2011 apuntan a una reducción del número de víctimas de violencia de género del $16 \%$ con respecto a 2010 , pasando de 73 a 61 mujeres 
asesinadas a manos de sus parejas o exparejas. A la espera de poder comparar estos datos con los del 2012, vuelve a plantearse la violencia de género como un fenómeno fluctuante. Las causas -sociales, económicas, culturales, etc.-, que provocan su aumento o disminución son por ello muy variadas y complejas, y por tanto muy difíciles de individualizar y aislar para poder ser erradicadas con éxito de la sociedad.

\section{ANÁLISIS Y RECEPCIÓN DE LAS CAMPAÑ̃S.}

\subsection{METODOLOGÍA DE LA INVESTIGACIÓN.}

La investigación que se presenta es de corte cuantitativo ya que cuenta con datos empíricos, objetivos y extrapolables y cuantitativos. Está investigación se encuentra en fase de pilotaje por lo que se ha realizado un estudio pretest con pocos sujetos ya que se pretende evaluar si el cuestionario es correcto o no y si alguna de las preguntas presenta alguna inconsistencia interna. Para confirmar este aspecto de la investigación todas las preguntas se han sometido a la prueba de fiabilidad interna utilizando para ello el parámetro estadístico Alfa de Cronbach (Igartua, 2006).

El cuestionario contiene escalas autoaplicadas previamente utilizadas en otras investigaciones -algunas de las cuales fueron adaptadas- y escalas elaboradas para la ocasión.

\subsection{OBJETIVOS E HIPÓTESIS DE LA INVESTIGACIÓN.}

El objetivo principal de esta investigación es conocer la percepción del público de las campañas realizadas por el Ministerio de Igualdad sobre la prevención de la violencia de género en el período 2008-2011. Se trata de conocer el mensaje percibido por parte del público, así como su valoración.

- Hipótesis 1: Se espera encontrar una valoración positiva de las campañas realizadas para la prevención de la violencia de género, esto es, se espera que las campañas sean valoradas positivamente por parte del público receptor.

- Hipótesis 2: Se espera encontrar una valoración positiva sobre la conveniencia de realizar campañas de violencia de género, esto es, se espera que los sujetos valoren positivamente la realización de este tipo de campañas para prevenir la violencia de género.

- Hipótesis 3: Se espera que los encuestados se identifiquen con las campañas realizadas, esto es, se espera que se pongan en el lugar del protagonista de la campaña y que sientan y piensen como ellos.

- Hipótesis 4: Se espera encontrar diferencias en la valoración de las diferentes campañas, esto es, se espera que los receptores valoren de manera diferente las campañas realizadas en el período objeto de estudio. 
Una vez analizados los resultados sobre valoración de las diferentes campañas se estudiará el contenido de cada una de ellas para poder obtener unas pautas que permitan desarrollar estrategias publicitarias más efectivas y que logren una mayor identificación entre el anuncio y los receptores y en especial, las víctimas de violencia de género.

\subsection{PARTICIPANTES EN LA FASE PILOTO DEL CUESTIONARIO.}

Para poder realizar esta fase de pilotaje se contó con la ayuda de algo más de una veintena de participantes quienes completaron el cuestionario realizado. Sobre los participantes, se buscaba una muestra no demasiado amplia para poder probar la consistencia interna del cuestionario, de cara a realizar un estudio que contase con un mayor número de participantes. Esta práctica es habitual en las investigaciones experimentales ya que se debe de comprobar la validez del cuestionario. Se quería que la muestra, a pesar de ser poco numerosa y representativa en cuanto a criterios cuantitativos se refiere, sí fuese una muestra representativa en cuanto a criterios cualitativos. Se buscó que el número de mujeres y hombres fuese equilibrado y que además fuesen de distintos entornos sociales, laborales y económicos. Se buscaba un perfil de muestra lo más significativo posible y heterogéneo dado que las campañas realizadas por las instituciones se dirigen a todo el público en general, no fragmentando su público objetivo. Este fue, por tanto, el criterio principal llevado a cabo para seleccionar la muestra de trabajo.

Al comienzo del cuestionario, y con el fin de obtener datos sociodemográficos básicos, se les pedía a los participantes que completaran una serie de preguntas: género, edad, ámbito geográfico, nivel de estudios y profesión. A modo de resumen, todos los encuestados viven en un entorno urbano $(\mathrm{M}=2, \mathrm{DT}=.0)^{4}$, son mayoría de mujeres $(\mathrm{M}=1.55, \mathrm{DT}=.51)$, con edades comprendidas entre los $26-40$ años $(\mathrm{M}=2.59$, $\mathrm{DT}=1,00)$, con estudios superiores $(\mathrm{M}=1.64, \mathrm{DT}=1.13)$. A continuación, se muestran unas tablas resúmenes de cada una de las variables sociodemográficas preguntadas a los encuestados expresadas en sujetos por caso y porcentaje.

Tabla 1. Género de los participantes

\begin{tabular}{|c|c|c|}
\cline { 2 - 3 } \multicolumn{1}{c|}{} & $\mathbf{N}^{\mathbf{0}}$ casos & Porcentaje \\
\hline Hombre & 10 & 45,5 \\
\hline Mujer & 12 & 54,5 \\
\hline Total & $\mathbf{2 2}$ & $\mathbf{1 0 0 , 0}$ \\
\hline
\end{tabular}

${ }^{4}$ A partir de ahora, M significará Media y DT Desviación típica. 
Tabla 2. Edad de los participantes

\begin{tabular}{|c|c|c|}
\cline { 2 - 3 } \multicolumn{1}{c|}{} & N Casos & Porcentaje \\
\hline $16-25$ & 3 & 13,6 \\
\hline $26-40$ & 8 & 36,4 \\
\hline $41-55$ & 6 & 27,3 \\
\hline $56-70$ & 5 & 22,7 \\
\hline Total & $\mathbf{2 2}$ & $\mathbf{1 0 0 , 0}$ \\
\hline
\end{tabular}

Tabla 3. Lugar en el que viven los participantes

\begin{tabular}{|l|c|c|}
\cline { 2 - 3 } \multicolumn{1}{c|}{} & N casos & Porcentaje \\
\hline Urbano & 22 & 100,0 \\
Rural & 0 & 0 \\
Total & $\mathbf{2 2}$ & $\mathbf{1 0 0 , 0}$ \\
\hline
\end{tabular}

Tabla 4. Nivel de estudios de los participantes

\begin{tabular}{|l|c|c|}
\cline { 2 - 3 } \multicolumn{1}{c|}{} & N casos & Porcentaje \\
\hline Titulado Superior & 16 & 72,7 \\
\hline Grado medio & 1 & 4,5 \\
\hline Bachillerato /FP & 2 & 9,1 \\
\hline Graduado & 3 & 13,6 \\
\hline Total & $\mathbf{2 2}$ & $\mathbf{1 0 0 , 0}$ \\
\hline
\end{tabular}

\subsection{DISEÑO DEL PROCEDIMIENTO DEL CUESTIONARIO Y DEL ESTUDIO.}

La investigación tiene como base un cuestionario que contiene escalas autoaplicadas $^{5}$. A continuación se describen en profundidad cada una de las preguntas y escalas utilizadas en la investigación. Al inicio del cuestionario además de especificar las indicaciones que debían tener en cuenta los encuestados a la hora de cumplimentarlo, se incluía información sociodemográfica básica -edad, sexo, ámbito geográfico, nivel de estudios y profesión-.

\footnotetext{
${ }^{5}$ La encuesta se mandó por correo electrónico a los encuestados quienes debieron completar y reenviarla a los investigadores por el mismo medio. Se enviaron a 30 personas diferentes y se recibieron 22 encuestas válidas. Algunas debieron de ser invalidadas ya que no habían sido completadas correctamente.
} 
La primera parte del cuestionario constaba de 12 preguntas en las que se preguntaba sobre la violencia de género y las campañas de prevención en general. Todas eran preguntas cerradas en las que la respuesta era dicotómica -sí o no- o una escala Likert formada por 5 ítems de respuesta.

La segunda parte del cuestionario analizaba cada una de las campañas de género realizadas por el Gobierno desde 2008 hasta 2011. El encuestado debía ver cada una de las campañas -se adjuntan los links- y valorar, mediante una escala Likert de 11 ítems, en qué medida les había gustado la campaña.

Además, debían contestar a una escala que mide la elaboración cognitiva o reflexión realizada durante el visionado. Esta escala, elaborada por Igartua (2007), está formada por 5 ítems en los que se evalúa en qué medida durante el visionado del spot el individuo "ha reflexionado sobre el tema que trataba", "ha pensado sobre la situación y las motivaciones de los personajes", "ha intentado ver cómo el argumento de la película se relacionaba con otros temas que me interesan", "ha pretendido sacar conclusiones sobre el tema central de la película" o bien "ha intentado no pensar demasiado sino distraerme y dejarme llevar por la película". El formato de respuesta es una escala de intensidad de cinco puntos, que van desde el 1 -nada- hasta el 5 mucho-.

A modo de conclusión, los encuestados debían valorar qué campaña les había gustado más y porqué. Así, se les pedía que señalaran cuál era el motivo por el que les había gustado más en función del: mensaje, imágenes, lema, identificación y real.

La consistencia interna del cuestionario es de $.663^{6}$, suficientemente alta para considerar que las escalas son fiables. Hay que señalar que algunas preguntas como la tercera - valora de 1 a 5 en qué medida son importantes las campañas de prevención de violencia de género (donde "1" significa que no son nada importantes y "5" que son muy importantes)-, cuarta -valora de 1 a 5 en qué medida son eficaces las campañas de prevención de violencia de género- y novena - ¿En qué medida crees que informar sobre violencia de género puede aumentar el número de agresiones o promover que estas se cometan?-, presentan fiabilidades muy bajas $(.402, .402, .010)$ por lo que deben de ser revisadas de cara a la investigación principal. Otras preguntas, como la primera - ¿Conoces a alguien que haya sufrido violencia de género?-, la quinta - En relación a la violencia de género, ¿en qué medida crees que son importantes las siguientes situaciones?- y la séptima - ¿Cuál de estas situaciones denunciarías si le sucedieran a un familiar, amigo, vecino...?-, entre otras, presentan fiabilidades muy altas $(.906, .859$ y .849$)$.

\footnotetext{
${ }^{6}$ Cuanto más se aproxime el valor alfa de Cronbach a su valor máximo, 1 , mayor es la fiabilidad de la escala. Además, en determinados contextos, se considera que valores del alfa superiores a 0,7 o 0,8 son suficientes para garantizar la fiabilidad de la escala.
} 


\subsection{RESULTADOS DE LA INVESTIGACIÓN.}

Una vez comprobada la fiabilidad de la encuesta, se analizaron los resultados de cada una de las preguntas, de esta manera se podrá conocer la opinión de los encuestados y comprobar si las hipótesis de partida se verifican o no.

La primera de las preguntas cuestionaba sobre si se conocía a alguien que hubiese sufrido violencia de género. La respuesta mayoritaria fue la de no $(M=1.73$, $\mathrm{DT}=.088$ ), que fue seleccionado por un $54,5 \%$ de las personas que realizaron la encuesta. A la pregunta cuyo enunciado indicaba sobre la necesidad de realizar campañas sobre prevención de violencia de género, un $100 \%$ de los encuestados respondió afirmativamente, aunque la uniformidad no se repite cuando se les pidió que valoraran de 1 a 5 -teniendo en cuenta que 1 significa que no son nada importantes y 5 que son muy importantes- cómo de importantes son las campañas. Un $9 \%$ señaló que no son nada importantes, un $40 \%$ muy importantes y un 31,8\% indicó que son importantes $(\mathrm{M}=3.82, \mathrm{DT}=1.25)$.

También se les preguntó si creían que las campañas son eficaces a la hora de prevenir la violencia de género $(\mathrm{M}=3.95$, $\mathrm{DT}=0.99)$. Un $40 \%$ de los encuestados señalan que son muy importantes, frente a un $4,5 \%$ que indica que no son nada importantes. Un 36\% indican que son importantes y un 18\% algo importantes-.

La quinta pregunta que contestaron los encuestados trataba de averiguar qué situaciones relacionadas con la violencia de género son más importantes. Las situaciones sobre las que se les preguntaba son: agresiones físicas $(M=4.86$, $\mathrm{DT}=.478)$; agresiones verbales $(\mathrm{M}=4.38, \mathrm{DT}=0.921)$; agresiones físicas y verbales $(\mathrm{M}=4.62$, $\mathrm{DT}=0.973)$ y maltrato psicológico $(\mathrm{M}=4.50$, $\mathrm{DT}=1.051)$. Un $86 \%$ de los encuestados señalaron que las agresiones físicas son muy importantes, un $77 \%$ las agresiones físicas y verbales, un $72 \%$ el maltrato psicológico y tan sólo un 59\% consideraron muy importante las agresiones verbales.

Otra cuestión que se les preguntó a los encuestados fue qué denunciarían si les sucediesen a ellos las siguientes situaciones: agresiones físicas $(\mathrm{M}=1.00, \mathrm{DT}=.00)$; agresiones verbales $(\mathrm{M}=1.30, \mathrm{DT}=.470)$; agresiones físicas y verbales $(\mathrm{M}=1.05$, $\mathrm{DT}=0.213)$ y maltrato psicológico $(\mathrm{M}=1.35, \mathrm{DT}=.489)$. Un $100 \%$ de los encuestados denunciarían agresiones físicas, un $63,6 \%$ las verbales, un $95,4 \%$ las físicas y verbales y un $59 \%$ denunciarían el maltrato psicológico.

Los datos difieren cuando se les pregunta a los encuestados qué situaciones denunciarían si le sucediera a un familiar, amigo, vecino... Los escenarios planteados son los mismos que en la pregunta anterior: agresiones físicas $(\mathrm{M}=1.14, \mathrm{DT}=.351)$; agresiones verbales $(\mathrm{M}=1.53, \mathrm{DT}=.513)$; agresiones físicas y verbales $(\mathrm{M}=1.14$, $\mathrm{DT}=0.351)$ y maltrato psicológico $(\mathrm{M}=1.35, \mathrm{DT}=.489)$. Un $86,3 \%$ denunciara una situación de agresiones físicas, un 40,9\% agresiones verbales, un 95,4\% agresiones 
físicas y verbales y un 59\% situaciones de maltrato psicológico. A la pregunta de si es necesario mostrar agresiones en las campañas de prevención de violencia de género para concienciar, un 59\% sí lo ven necesario $(\mathrm{M}=1.41$, DT=.503).

Un 36,3\% de los encuestados creen que informar sobre violencia de género no aumenta el número de agresiones $(\mathrm{M}=2.41, \mathrm{DT}=2.462)$, un $10 \%$ creen que aumenta algo y el resto de los encuestados, un $54,5 \%$, creen que no es un factor determinante.

Sobre si en estos momentos hay más casos de violencia de género, la opinión está dividida, ya que el $50 \%$ considera que sí frente al otro $50 \%$ que señala que no $(\mathrm{M}=1.50, \mathrm{DT}=.512)$. Aquellos que han señalado que sí, $(\mathrm{n}=11)$, creen un $36,3 \%$ tiene bastante que ver que se informe más en los medios ( $\mathrm{M}=3.40$, $\mathrm{DT}=1.350)$, un $54,5 \%$ creen que es porque las mujeres aguantan menos $(\mathrm{M}=3.40, \mathrm{DT}=1.350)$, un $36,3 \%$ señalan que no tiene que ver la crispación social $(\mathrm{M}=3.09$, DT=1.446), un 36,3\% indican que el cambio de mentalidad tiene mucho que ver $(\mathrm{M}=3.64, \mathrm{DT}=1.433)$ y un $54,5 \%$ creen que tiene bastante que ver que ahora se denuncia antes $(\mathrm{M}=3.45, \mathrm{DT}=$ $1.128)$.

La última pregunta de este bloque trataba de averiguar en qué medida son adecuadas las medias de protección a las víctimas $(\mathrm{M}=2.95, \mathrm{DT}=1.174)$ en una escala de 1 -no son adecuadas- a 5 -son muy adecuadas-. Un 36,3\% las consideraba adecuadas, frente a un $4 \%$ que las consideraba nada adecuadas y un $13,6 \%$ muy adecuadas.

La segunda parte del cuestionario estaba centrada en las diferentes campañas realizadas por el Gobierno para prevenir la violencia de género. Según los encuestados $(n=22)$, la campaña que más les ha gustado es la del 2010 con una media de 7.59 (DT=1.83), seguida de la campaña del $2008(\mathrm{M}=7.45$, DT=1.56) y 2007 $(\mathrm{M}=7.14, \mathrm{DT}=2.58)$. La campaña que menos ha gustado a los encuestados es la del 2009, con una media bastante baja, de 5.80 (DT=2.48).

Tabla 5. Elaboración cognitiva realizada por los participantes durante las diferentes campañas

3. 6. RESULTADOS DE LA ENCUESTA.

\begin{tabular}{|c|c|c|c|c|c|c|c|c|}
\hline & \multicolumn{2}{|c|}{$\begin{array}{l}\text { Campaña } \\
2008\end{array}$} & \multicolumn{2}{|c|}{$\begin{array}{l}\text { Campaña } \\
2009\end{array}$} & \multicolumn{2}{|c|}{$\begin{array}{l}\text { Campaña } \\
2010\end{array}$} & \multicolumn{2}{|c|}{$\begin{array}{l}\text { Campaña } \\
2011\end{array}$} \\
\hline & $\mathbf{M}$ & $T^{\text {D }}$ & $\mathbf{M}$ & DT & $\mathbf{M}$ & $T^{\text {D }}$ & $\mathbf{M}$ & $T^{\mathbf{D}}$ \\
\hline $\begin{array}{l}\text { He } \\
\text { reflexionado sobre } \\
\text { el tema en que } \\
\text { trataba }\end{array}$ & $\begin{array}{l}3, \\
86\end{array}$ & $\begin{array}{c}0, \\
793\end{array}$ & ${ }^{3} 8^{3}$ & $\begin{array}{l}1,0 \\
78\end{array}$ & 45 & $\begin{array}{c}1, \\
050\end{array}$ & $\begin{array}{r}4 \\
, 10\end{array}$ & $68^{, 7}$ \\
\hline
\end{tabular}




\begin{tabular}{|c|c|c|c|c|c|c|c|c|}
\hline \begin{tabular}{l}
\multicolumn{1}{c}{ He pensado } \\
sobre la situación \\
y las motivaciones \\
de los personajes
\end{tabular} & $\begin{array}{r}3 \\
62\end{array}$ & $\begin{array}{r}1, \\
024\end{array}$ & $\begin{array}{r}3 \\
, 57\end{array}$ & $\begin{array}{l}1,1 \\
65\end{array}$ & 20 & $\begin{array}{r}1, \\
152\end{array}$ & $\begin{array}{r}4 \\
, 10\end{array}$ & $68^{, 7}$ \\
\hline $\begin{array}{l}\quad \text { He intentado } \\
\text { ver cómo el } \\
\text { argumentado de la } \\
\text { película se } \\
\text { relacionaba con } \\
\text { otros temas }\end{array}$ & 24 & $\begin{array}{c}1, \\
136\end{array}$ &, $43^{2}$ & $\begin{array}{l}1,1 \\
65\end{array}$ & $\begin{array}{c}2, \\
10\end{array}$ & $\begin{array}{c}1 \\
210\end{array}$ & $\begin{array}{r}{ }^{2} \\
, 62\end{array}$ & $\begin{array}{r}1, \\
244\end{array}$ \\
\hline $\begin{array}{l}\text { He pretendido } \\
\text { sacar conclusiones } \\
\text { sobre el tema } \\
\text { central de la } \\
\text { película }\end{array}$ & 43 & $\begin{array}{c}1, \\
028\end{array}$ & ${ }^{3}$ & $22^{1,3}$ & $\begin{array}{c}3, \\
11\end{array}$ & $\begin{array}{r}1, \\
370\end{array}$ & $\begin{array}{r}3 \\
, 55\end{array}$ & $\begin{array}{c}1, \\
146\end{array}$ \\
\hline $\begin{array}{l}\quad \text { He intentado } \\
\text { no pensar } \\
\text { demasiado sino } \\
\text { distraerme y } \\
\text { "dejarme llevar" } \\
\text { por la película }\end{array}$ & $\begin{array}{l}2, \\
91\end{array}$ & $\begin{array}{r}4, \\
669\end{array}$ & $\begin{array}{c}2 \\
, 14\end{array}$ & $\begin{array}{l}1,4 \\
90\end{array}$ & $\begin{array}{c}2, \\
14\end{array}$ & $\begin{array}{r}1, \\
424\end{array}$ & $\begin{array}{r}2 \\
, 10\end{array}$ & $\begin{array}{c}1, \\
375\end{array}$ \\
\hline
\end{tabular}

\subsubsection{HIPÓTESIS 1.}

La primera de las hipótesis espera encontrar una valoración positiva de las campañas realizadas para la prevención de la violencia de género, esto es, se espera que las campañas sean valoradas positivamente por parte del público receptor.

La primera de las hipótesis se ha visto contrastada positivamente ya que el $100 \%$ de los encuestados valoran positivamente la realización de campañas de prevención de violencia de género. Además, todas las campañas analizadas en este estudio han sido aprobadas por los encuestados no habiendo valorado negativamente ninguna de ellas.

\subsubsection{HIPÓTESIS 2.}

La segunda de las hipótesis espera encontrar una valoración positiva sobre la conveniencia de realizar campañas de violencia de género, esto es, se espera que los sujetos valoren positivamente la realización de este tipo de campañas para prevenir la violencia de género. 
Esta segunda hipótesis también se ha visto reafirmada por los encuestados, tal y como se puede ver en la hipótesis primera. Además, un $40 \%$ de los encuestados indicó que son muy importantes y un 31,8\% indicó que son importantes. Tan sólo un $9 \%$ de los encuestados señaló que no son nada importantes. Por lo tanto, esta segunda hipótesis también ha sido contrastada positivamente.

\subsubsection{HIPÓTESIS 3.}

La tercera de las hipótesis espera que los encuestados se identifiquen con las campañas realizadas, esto es, se espera que se pongan en el lugar del protagonista de la campaña y que sientan y piensen como ellos.

Tal y como se puede ver en la tabla 5 , los encuestados han reflexionado sobre la violencia de género mientras veían las campañas. Con la que más han reflexionado es con la campaña del $2011(\mathrm{M}=4.10, \mathrm{DT}=.0768)$ y con la que menos con la del 2010 $(\mathrm{M}=3.45, \mathrm{DT}=1.050)$. Análoga situación se produce con la afirmación "he pensado sobre la situación y las motivaciones de los personajes" ya que es la campaña del 2011 la que presenta medias más altas $(\mathrm{M}=4.10, \mathrm{DT}=.0768)$ y la del 2011 bajas $(\mathrm{M}=3.20$ y $\mathrm{DT}=1.152)$. La afirmación "he intentado ver cómo el argumentado de la película se relacionaba con otros temas" obtiene unas medias más bajas en todas las campañas, aunque se vuelve a repetir que la campaña del 2011 tenga medias más altas $(\mathrm{M}=2.62, \mathrm{DT}=1.244)$ y la del 2010 más bajas $(\mathrm{M}=2.10, \mathrm{DT}=1.210)$.

La campaña del 2009 ha obtenido medias más altas $(\mathrm{M}=3.62$, $\mathrm{DT}=1.322)$ en la afirmación He pretendido sacar conclusiones sobre el tema central de la película y la del 2010, de nuevo, las más bajas $(\mathrm{M}=3.11$, $\mathrm{DT}=1.370)$. La última de las afirmaciones, "he intentado no pensar demasiado sino distraerme y "dejarme llevar" por la película", ha obtenido valoraciones bajas lo cual es positivo ya que significa que los encuestados se han centrado en el mensaje de las campañas y han reflexionado sobre el mismo durante el visionado. La campaña del 2009 es la que ha conseguido una mayor puntuación $(\mathrm{M}=2.91, \mathrm{DT}=4.669)$ por lo que los encuestados se han distraído más y con la que menos se habrían distraído es con la campaña del 2011 $(\mathrm{M}=2.10, \mathrm{DT}=1.375)$. A tenor de estos resultados, se podría afirmar que es la última de las campañas, la del 2011, la que consigue que los receptores reflexionen más durante el visionado.

\subsubsection{HIPÓTESIS 4.}

La cuarta de las hipótesis espera encontrar diferencias en la valoración de las diferentes campañas, esto es, se espera que los receptores valoren de manera diferente las campañas realizadas en el período objeto de estudio.

A la vista de los resultados obtenidos, se puede afirmar que la última de las hipótesis planteadas en la investigación ha sido contrastada positivamente ya que no todas las campañas han sido valoradas del mismo modo por los encuestados. Tal y 
como se ha visto con anterioridad, la campaña que más les ha gustado es la del 2010 con una media de 7.59 ( $\mathrm{DT}=1.83)$, seguida de la campaña del $2008 \quad(\mathrm{M}=7.45$, $\mathrm{DT}=1.56)$ y $2007(\mathrm{M}=7.14$, $\mathrm{DT}=2.58)$. La campaña que menos ha gustado a los encuestados es la del 2009, con una media bastante baja, de $5.80(\mathrm{DT}=2.48)$. Así pues, la campaña cuyo lema era "Saca tarjeta roja al maltratador" cuyo objetivo era concienciar a la población es la que mejor valoración ha obtenido frente a la campaña del 2009 -"ante el maltrato, todos y todas una"- cuyo objetivo era concienciar no sólo a la sociedad sino a las mujeres en particular.

\section{CONCLUSIONES}

Teniendo en cuenta estos primeros datos obtenidos, se ha procedido a realizar una valoración de estas campañas de sensibilización, comparando estos datos con los resultados estadísticos vinculados a la violencia de género en España, publicadas por el Ministerio de Sanidad, Igualdad y Políticas Sociales. Para conocer la eficacia de estas campañas es necesario un control exhaustivo de su grado de penetración en la población. El control más operativo que puede realizarse consiste en evaluar la eficacia y transcendencia de la campaña entre la población objetivo al finalizar la misma. Si en dicho control se efectúa un seguimiento de la penetración, es posible que se pueda determinar el nivel de presión a partir del cual ya no se produce un incremento, lo que permite adoptar decisiones en campañas futuras sobre la duración de las mismas y los niveles de inversión más convenientes.

La medición de la eficacia de las campañas en una primera etapa conlleva un control del tratamiento de diferentes entidades y personas que actúan a lo largo del proceso y deben colaborar activamente para demostrar la calidad y la utilidad de las campañas difundidas. La segunda etapa sirve para evaluar los resultados obtenidos en los cambios de actitudes, con los que se puede establecer hasta qué punto la campaña ha permitido modificar las percepciones de las personas en relación a este problema. Por último, existe un tercer nivel en el que se miden los cambios en el comportamiento de las personas generados a raíz de la campaña.

El análisis apunta a que son precisamente los spots televisivos las herramientas más eficaces en la concienciación contra la violencia de género, en cuanto que son éstos los que mayor proyección y calado social tienen. Según la encuesta del CIS sobre la Percepción de la Violencia contra la Mujer en España (Informe Ejecutivo del III Informe Anual del Observatorio Estatal de Violencia sobre la Mujer, 2010, 10), el $70,8 \%$ de la población afirma conocer la información relativa a violencia de género a través de la televisión. Se trata de un aplastante dominio del medio televisivo sobre el resto de los medios de comunicación de masas. Si a este dato unimos el que la televisión es también, para el $18,9 \%$ de la población el segundo medio de comunicación del que procede la información sobre violencia de género, arroja una 
cifra global del $89,7 \%$ de la sociedad española que conoce la violencia de género a través del medio televisivo. Ello incluye no solo las campañas de concienciación y sensibilización sobre este problema, sino también noticias y reportajes sobre la violencia contra la mujer.

A partir de 2008, coincidiendo con el momento en que socialmente la violencia machista sufría un repunte en la percepción del problema en la población, se aumentan exponencialmente los recursos y medios al servicio de estas campañas de sensibilización televisivas. A pesar de ello, la mayoría de la población opina que éstas no determinan su estado de opinión, en concreto un 58,3\% (Informe Ejecutivo, 2010: $10)$.

Analizados estos datos, se obtienen los siguientes resultados:

- La mayoría de la población no considera a las campañas en televisión como un elemento que varíe su forma de pensar sobre este tema $(58,3 \%)$.

- La violencia de género es considerada tan solo por el $2,7 \%$ de la población como uno de los tres problemas más graves de España (dato de la encuestas del CIS de diciembre de 2009).

- A modo de reflexión, el aumento del número de víctimas durante 2010 en más de un $23 \%$ respecto a 2009 , a pesar de que los medios y recursos al servicio de la lucha contra la violencia de género aumentaron de forma exponencial, obliga a preguntarse hasta qué punto estas campañas sensibilizadoras son realmente efectivas. Quizás existe un desequilibrio entre la cantidad de recursos disponibles y la penetración del mensaje, desequilibrio que los organismos e instituciones responsables de la erradicación de la violencia de género, deberían tratar de solucionar.

Ante la perspectiva de que la violencia de género es un fenómeno que fluctúa, cambia, aparecen nuevos condicionantes y desaparecen otros, la campaña en televisión debe ser lo suficientemente flexible para poder variar sus objetivos y por ello sus contenidos y mensajes si la situación del problema y la percepción social lo hacen necesario. Si dicha campaña pierde validez o importancia, por la causa que sea, las instituciones deberían ser capaces de reaccionar y actualizarla, o realizar una nueva campaña. Dado que en el estudio realizado, el 100\% de los encuestados valoran positivamente la realización de campañas de prevención de violencia de género para poder concienciar sobre el problema el Gobierno no debería desatender esta vía, ya que éstas son valoradas de manera muy positiva por la ciudadanía, tal y como se ha visto en el estudio realizado, donde un $40 \%$ valoraban que son importantes. Quizá la vía a utilizar es la de protagonizar las campañas con personas relevantes de la sociedad ya que, según el estudio realizado, ésta es la campaña que mayor valoración 
ha obtenido. Quizá, ver a personas conocidas, en lugar de personas anónimas, ayude a los espectadores y a los ciudadanos a valorar de diferente manera el problema de la violencia de género.

\section{BIBLIOGRAFÍA}

GARCÍA, MARIOLA (2000): Las claves de la publicidad. Madrid: ESIC

GONZÁLEZ, JUAN ANTONIO (1996): Teoría general de la publicidad. Madrid: FCE

IGARTUA, JUAN JOSÉ (2006): Métodos cuantitativos en comunicación. Barcelona: Editorial Bosch.

---- (2007): Persuasión Narrativa. El papel de la identificación con los personajes a través de las culturas. Alicante: Editorial Club Universitario.

---- y HUMANES, MARIA LUISA (2004): Teoría e investigación en comunicación social. Madrid: Editorial Síntesis.

LÓPEZ, PILAR (dir.) (2005): "Representación de Género en los Informativos de Radio y Televisión". Madrid, Instituto Oficial de Radio y Televisión, en http://www.rtve.es/oficial/iortv/Dossier\%20MUJER\%20Y\%20VIOLENCIA.pd f (consultado el 21/11/2010)

MARTINEL, EMMA. (1979): "Lingüística y Publicidad", Revista española de lingüística, Año ${ }^{\circ}$ 9, Fasc. 1, Págs. 173-190

MOLINA, ALICIA. (dir.) (2005): Análisis de las campañas publicitarias de prevención de la violencia contra la mujer en España (2005). Valencia, en

http://www.valencia.es/mujer/mujer.nsf/0/02786D44FC3CC715C1257404002C24

BA/\$FILE/17894744.pdf?OpenElement\&=lang=1, (consultado el 20/12/2011)

ROMAN, KENNETH.; MAAS, JANE (1995): El nuevo como anunciar. Madrid, Grupo Control.

ROMERO, MARIA VICTORIA, BETÉS, KETY (2005): Lenguaje publicitario: la seducción permanente. Barcelona: Ariel Comunicación

VELLÓN, JAVIER (2007): Estrategias lingüisticas de los textos publicitarios. Barcelona: OUC.

Datos estadísticos relativos a violencia de género en España.

http://www.migualdad.es/ss/Satellitec=MIGU_Multimedia_FP\&cid=11930498868 48\&language $=$ cas_ES\&page

$=1193049890202$ \&pagename=MinisterioIgualdad/MIGU_Multimedia_FP/MIGU _listadoSubcategoria

(consultado el 23/01/2011) 


\section{Vídeos}

Videos Campañas contra la Violencia de Género 2008:

http://www.migualdad.es/ss/Satellite?c=MIGU_Campania_FA\&cid=11930498325 42\&pagename=MinisterioIgualdad/MIGU_Campania_FA/MIGU_campaniaGenerica, (consultado el 12/01/2011);

Videos Campaña contra la Violencia de Género (2009):

http://www.migualdad.es/ss/Satellite?c=MIGU_Campania_FA\&cid=12446473924 75\&language $=$ cas_ES\&pageid $=1193049831625$ \&pagename $=$ Ministeriolgualdad $/ \mathrm{MI}$ GU_Campania_FA/MIGU_campaniaGenerica (consultado el 12/01/2011)

Videos Campaña contra la Violencia de Género (2010):

http://213.27.203.194/ss/Satellite?c=MIGU_Campania_FA\&cid=1244647754921 \&language $=$ cas_ES\&pageid $=1193049831625 \&$ pagename $=$ MinisterioIgualdad $/$ MIGU _Campania_FA/MIGU_campaniaGenerica (consultado el 12/01/2011)

Videos Campaña contra la Violencia de Género (2011):

http://www.seigualdad.gob.es/secretaria/acciones/campanas/eligeVivir.htm (consultado el 20/12/2011)

I Informe Anual del Observatorio Estatal sobre Violencia contra la Mujer. (2007). Ministerio de Igualdad Social de España. www.migualdad.es (consultado el 12/01/2011)

II Informe Anual del Observatorio Estatal sobre Violencia contra la Mujer. (2009). Ministerio de Igualdad Social de España.www.migualdad.es (consultado el $12 / 01 / 2011)$

III Informe Anual del Observatorio Estatal sobre Violencia contra la Mujer. Informe Ejecutivo. (2010) Ministerio de Igualdad Social de España.

http://www.migualdad.es/ss/Satellite?blobcol=urldata\&blobheader=application/pd f\&blobheadername $1=$ Content -

disposition\&blobheadervalue $1=$ inline $\&$ blobkey $=$ id\&blobtable $=$ MungoBlobs\&blobwh ere $=1244653592435 \&$ ssbinary $=$ true (consultado el 23/01/2011)

IV Informe Anual del Observatorio Estatal sobre Violencia contra la Mujer. Informe Ejecutivo (2011). Ministerio de Igualdad Social de España. (consultado el 20/12/2011)

http://www.seigualdad.gob.es/secretaria/cifrasInformes/infIgualdad.htm 